\title{
THE RAPID SEPARATION OF HEROIN AND MORPHINE.
}

BY JAMES M. DORAN.

The separation of heroin and morphine when present in solution in small amounts has caused the writer some difficulty in times past, not only in making quantitative determinations of each, but even in determining their presence qualitatively.

This separation becomes of peculiar importance because of the fact that the act of Congress, approved December 17, 1914, popularly known as the Harrison Anti-Narcotic Law, provides in Section 6, among other things, for the exemption of certain preparations cantaining not more than one-fourth of a grain of morphine nor more than one-eighth of a grain of heroin per fluidounce, or if solid or semisolid in one avoirdupois ounce, provided such preparations are in fact medicines and not preparations for the purpose of evading the intentions and provisions of the act.

Many preparations have been examined in the laboratory of the Bureau of Internal Revenue which have been found to contain both morphine and heroin, besides other alkaloids, and in many cases in amounts very close to the exemption limit.

The usually-accepted method of separating heroin and morphine is based upon the solubility of the free base heroin, from an ammoniacal solution, in ether, morphine being relatively insoluble and remaining in the aqueous solution. Experience with this procedure has not been very satisfactory. The ordinary ether which contains up to four percent of ethyl alcohol carries morphine with it on account of the solubility of the free base morphine in ethyl alcohol.

The result is that the presence of morphine in the heroin extraction not only vitiates the quantitative result, but, in the case of very small amounts of heroin being present, the qualitative detection is rendered very difficult, if not entirely impossible. The use of absolute ether did not prove any more satisfactory. The heroin extraction contained some morphine, possibly due to the solution of some of the aqueous solution containing the free morphine in the ether.

In addition to these factors and possibly some others, objection is also made to the necessity of four or five extractions to get out all of the heroin, and the sources of error due to the necessity of drawing off the aqueous solution and transferring back to the separatory funnel after each extraction.

Any use of an extraction method in which a fixed alkali hydroxide is used to free the alkaloid base and hold the morphine in solution as alkali morphinate is valueless so far as the heroin is concerned, as it is almost immediately hydrolyzed into morphine by splitting off of the diacetyl radicle.

The problem seemed to be to find a solvent which would extract the heroin from the morphine in a fairly pure state and leave the morphine in such condition that it could be determined later. Chemically pure carbon tetrachloride proved to be such a solvent, and some of the details of its use are presented here. It dissolves the free base heroin very readily, and, while morphine is dissolved by it very slightly, the amount of solvent used is small and the length of time required for the solution of the heroin is so short that the amount of morphine taken up by the carbon tetrachloride is negligible.

In the experimental work no morphine was found in the heroin residue. Settling of the solvent is rapid, owing to its high specific gravity, and there is practically no emulsion formed. 
'The advantage of an immiscible solvent with these properties is obvious when a number of determinations are to be made.

Briefly the method used for separating and determining heroin and morphine is as follows:

Take of the sample a portion representing not over 0.2 gramme of either heroin or morphine: if tablets, dissolve in water slightly acidified with hydrochloric acid; if solid matter, treat with water acidified with dilute $\mathrm{HCl}$ and filter; if an alcoholic solution, make very slightly acid with dilute $\mathrm{HCl}$ and evaporate off the alcohol before proceeding.

Transfer the solution of the salts of the alkaloids to a separatory funnel, preferably of the Squibb type, neutralize with $\mathrm{NH}_{4} \mathrm{OH}$ and make alkaline with 6 to 8 drops of $\mathrm{NH}_{4} \mathrm{OH}$ ( 1 to 1 ). Add $25 \mathrm{Cc}$. $\mathrm{CCl}_{4}$, shake, let stand, and draw off through a $7-\mathrm{cm}$. dry filter paper into a tared dish. Repeat the extraction twice, making three extractions in all. Evaporate on steam-bath to dryness, heat in oven at $100^{\circ} \mathrm{C}$. for not over 10 minutes, cool, and weigh as heroin. The amount of heroin may be further checked by taking up in an excess of $\mathrm{N} / 25 \mathrm{H}_{2} \mathrm{SO}_{4}$, heating to effect complete solution, and titrating the excess of acid with $N / 25$ $\mathrm{NaOH}$, using methyl red as an indicator.

The weighed residue of heroin can be tested qualitatively if desired.

It is best done by taking it up in about $1 \mathrm{Cc}$. of $\mathrm{N} / 10 \mathrm{HCl}$, placing a drop of this solution on a slide, and adding a drop of 5 percent platinic chloride solution. An amorphous yellow precipitate is formed, and, when viewed under a microscope, very characteristic crystals of the heroin platinic chloride appear within a few minutes. This crystal form has been described by Putt, ${ }^{1}$ and is to be recommended as an excellent identification of the alkaloid heroin. Color tests may also be made on the residue, using Froehde's and Marquis's reagents.

For the determination of the morphine remaining as the free base in the ammoniacal solution the iso-butyl alcohol-chloroform extraction method of Dohme $^{2}$ is recommended. It has been found to be quick, satisfactory as to manipulation, and to remove practically all of the morphine. The procedure is as follows: The ammoniacal morphine solution remaining after the heroin has been removed is extracted with about $30 \mathrm{Cc}$. of iso-butyl alcohol-chloroform ( 1 to 1 ) in the same separatory funnel, and the extract drawn off through a dry $7-\mathrm{cm}$. filter paper into a thick-walled flask. This extraction is repeated until the morphine has been removed from the mother liquor; three extractions will usually suffice. The flask is placed on a steam-bath and the $\mathrm{CHCl}_{3}$ distilled off. When the $\mathrm{CHCl}_{3}$ has been driven off, suction is applied and the iso-butyl alcohol is distilled off under diminished pressure. An intermediate suction flask serves, to condense the iso-butyl alcohol, which may be recovered for use again by a redistillation. The residue of morphine is taken up with an excess of $\mathrm{N} / 25 \mathrm{H}_{2} \mathrm{SO}_{4}$, heated to insure complete solution, and the excess titrated with $\mathrm{N} / 25 \mathrm{NaOH}$, using methyl red as an indicator.

For the qualitative detection of morphine, in addition to the well-known color reactions, the characteristic crystals of morphine precipitated with iodine, as seen under the microscope, are very distinctive, and the reader is referred to Putt (see above) for further details of the test. The writer's experience has been that 15 percent of morphine present vitiates the heroin platinic chloride crystals test, and, vice versa, the presence of 50 percent of heroin prevents the formation of the morphine-iodine crystals. These facts, together with the well-known weaknesses

J. I. and E. C., vol. 4, No. 7.

Jour. A. Ph. A., vol. iv, No. 1, p. 85. 
of the color reactions for identification of these alkaloids when not in a pure state, emphasize the necessity for obtaining fairly pure residues even for qualitative detection.

In the experimental work the salts used, morphine sulphate, heroin, and diacetyl morphine hydrochloride, were the ordinary commercially pure salts as sold by the trade, and no attempt was made to further purify them. Commercial morphine sulphate, while it is considered a fairly pure commercial product, nevertheless contains a small amount of the other alkaloids of opium, principally codeine. This fact probably accounts for some of the discrepancies of the analyses other than those due to analytical errors.

Following are some of the results obtained:

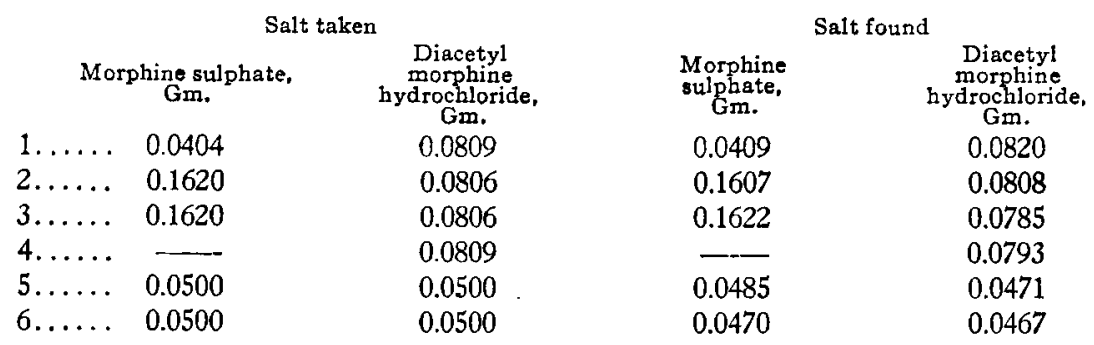

In titrating the heroin residue and using the factor $1 \mathrm{Cc} . \mathrm{N} / 25 \mathrm{H}_{2} \mathrm{SO}_{4}=0.01622$ $\mathrm{Gm}$. heroin hydrochloride or diacetyl morphine hydrochloride, which was the salt used, it was observed that the result was about 5 percent below the weight of the residue if calculated as the hydrochloride, and also bore the same relation to the amount of the salt weighed out. This matter was taken up by Harris and Clover, ${ }^{3}$ who showed that the salt on the market was monohydrated. Shaefer ${ }^{4}$ is also quoted as stating that the only salt found on the market was monohydrated. This should be remembered if calculating the amount of commercial heroin hydrochloride present from the titration figures: $1 \mathrm{Cc} . \mathrm{N} / 25 \mathrm{H}_{2} \mathrm{SO}_{4}=0.01694 \mathrm{Gm}$. heroin hydrochloride, or diacetyl morphine hydrochloride, monohydrated.

Carbon tetrachloride readily dissolves the free bases of dionin (ethyl morphine), peronin (benzoyl morphine), and codeine (methyl morphine).

If codeine, heroin, and morphine are present, the codeine and heroin can be removed by $\mathrm{CCl}_{4}$ and weighed. The morphine can then be determined by the iso-butyl alcohol-chloroform extraction. Another portion of the solution may then be treated with $10 \mathrm{Cc}$. of $2 \mathrm{~N} \mathrm{NaOH}$, which completely hydrolyzes the heroin to morphine without the use of heat.

The codeine may be extracted from the alkaline solution by $\mathrm{CCl}_{4}$ and weighed, the alkaline liquor containing the other alkaloids is acidified, then made alkaline with $\mathrm{NH}_{4} \mathrm{OH}$, and the tatal morphine determined. The increase of morphine is due to heroin, and it may be calculated. However, any complex mixture of alkaloids, as is well known, requires special treatment, and the foregoing remarks apply only to the simple mixtures.

Two hours is ample to complete the determination of heroin and morphine as outlined above, and the procedure is well adapted to routine qualitative and quantitative examinations.

Laboratory, Bureau of Internal Revenue, Washington, D. C.

${ }^{8}$ Jour. A. Ph. A., vol. iv, No. 3, p. 291.

- Amer. Jour. Pharm., 82, p. 220. 